Please do not remove this page

RMIT

UNIVERSITY

\title{
Nonlinear Tamm states in periodic photonic structures
}

Rosberg, Christian; Neshev, Dragomir; Kartashov, Yaroslav; Vincencio, Rodrigo; Krolikowski, Wieslaw; Molina, Mario; Mitchell, Arnan

https://researchrepository.rmit.edu.au/esploro/outputs/9921862041901341/filesAndLinks?institution=61RMIT_INST\&index=null

Rosberg, C., Neshev, D., Kartashov, Y., Vincencio, R., Krolikowski, W., Molina, M., Mitchell, A., Vysloukh, V., Torner, L., \& Kivshar, Y. (2006). Nonlinear Tamm states in periodic photonic structures. Optics and Photonics News, 17(12), 29-29. https://doi.org/10.1364/OPN.17.12.000029

Document Version: Published Version

Published Version: https://doi.org/10.1364/OPN.17.12.000029

Repository homepage: https://researchrepository.rmit.edu.au

(c) 2006 Optical Society of America

Downloaded On 2023/04/26 23:30:00 +1000

Please do not remove this page 


\title{
Nonlinear Tamm States in Periodic Photonic Structures
}

\author{
Christian R. Rosberg, Dragomir N. Neshev, Yaroslav V. Kartashov, Rodrigo A. Vicencio, \\ Wieslaw Krolikowski, Mario I. Molina, Arnan Mitchell, Victor A. Vysloukh, Lluis Torner and Yuri S. Kivshar
}

B ack in 1932, the famous Russian scientist Igor E. Tamm predicted that a truncated crystalline lattice could support special types of electronic states that are bound at the very edge of the semi-infinite periodic potential. ${ }^{1}$ These states, known in many fields as Tamm states, represent a special class of spatially localized surface waves, which, in general, may appear at interfaces between different physical media. An optical analog of linear Tamm states has been described theoretically and demonstrated experimentally for an interface separating periodic and homogeneous dielectric materials. ${ }^{2}$ Despite the fact that many theoretical concepts have been successfully introduced in the physics of surface waves, nonlinear Tamm states have never been experimentally observed.

In a recent paper, we experimentally demonstrated self-action of a narrow laser beam propagating near the edge of a lithium niobate $\left(\mathrm{LiNbO}_{3}\right)$ waveguide array with defocusing nonlinearity and a semi-infinite periodic refractive index modulation in the transverse direc- tion, ${ }^{3}$ as shown schematically in part (b) of the figure. For the first time to our knowledge, we observed the formation of surface gap solitons or nonlinear Tamm states. While linear surface modes do not exist in this kind of system, light self-trapping is observed in the nonlinear regime above a certain threshold power when the propagation constant is shifted into the gap of the photonic transmission spectrum.

The detailed theory of such nonlinear Tamm states was developed a few months earlier by Kartashov et al. ${ }^{4}$ Encouraged by the recent studies of discrete surface solitons, ${ }^{5}$ they predicted the existence of surface gap solitons at the interface between a uniform dielectric medium and a photonic lattice with defocusing nonlinearity.

In such systems, light localization occurs in the form of nonlinear surface modes exhibiting a staggered phase structure, as shown in (a). The alternating phase of the beam tail inside the periodic medium reflects the fact that the propagation constant of the self-localized mode (a)

(b)

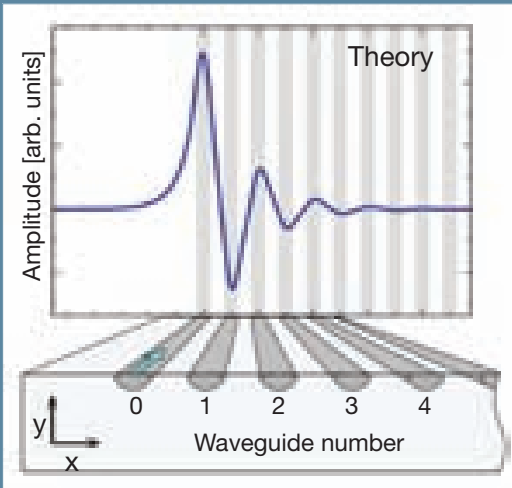

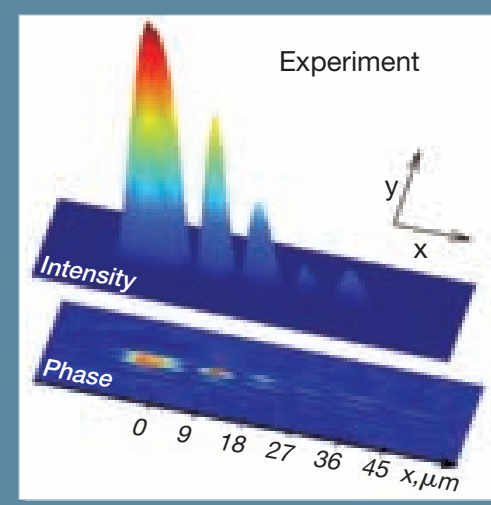

(c)

Theoretical prediction $(a, b)$ and experimental observation $(c, d)$ of nonlinear Tamm states in a truncated photonic lattice. (a) Theoretical profile of a nonlinear Tamm state-a surface gap soliton. ${ }^{4}$ (b) Schematic of the waveguide array geometry. (c) Three-dimensional representation of the nonlinear surface state observed above the localization threshold. ${ }^{3}$ The horizontal and vertical sample coordinates are $x$ and $y$, respectively. (d) Experimental plane-wave interferogram demonstrating the staggered phase structure of the nonlinear Tamm state.

lies within the photonic bandgap. This essential feature enables one to draw a direct analogy to the electronic Tamm states and extend this concept to the nonlinear regime, so that the surface gap solitons can be termed nonlinear Tamm states. They possess a unique combination of properties related to both electronic and optical surface waves and gap solitons.

Part (c) of the figure depicts a threedimensional representation of the spatial beam intensity distribution of a nonlinear Tamm state observed in experiment. ${ }^{3}$ The nonlinear mode was excited by injecting a narrow probe beam into the surface waveguide at the edge of the periodic structure. Part (d) shows the corresponding interference pattern created when superimposing an inclined plane reference wave in order to reveal the phase structure of the output beam. A half-period vertical shift of the interference fringes, corresponding to an exact $\pi$ phase jump in the horizontal beam direction, is clearly observed between each lattice site, as predicted by theory. ${ }^{4}$

The ability to generate these types of optical surface modes could lead to novel and effective experimental tools for the study of nonlinear effects near surfaces. $\triangle$

[ C.R. Rosberg, D.N. Neshev, W. Krolikowski and Y.S Kivshar are with CUDOS at the Australian National University, Canberra, Australia. Y.V. Kartashov and L. Torner are with ICFO, Barcelona, Spain. R.A. Vicencio is with the Max-Planck-Institut für Physik komplexer Systeme, Dresden, Germany. M.I. Molina is with the departamento de física, facultad de ciencias, Universidad de Chile, Santiago, Chile. A. Mitchell is with the School of Electrical \& Computer Systems Engineering, RMIT University, Melbourne, Australia. V.A. Vysloukh is with the departamento de física y matemáticas, Universidad de las Américas, Puebla, Mexico. ]

\footnotetext{
References

1. I.E. Tamm. Z. Phys. 76, 849 (1932).

2. P. Yeh et al. Appl. Phys. Lett. 32, 102 (1978).

3. C.R. Rosberg et al. Phys. Rev. Lett. 97, 083901 (2006).

4. Y.V. Kartashov et al. Phys. Rev. Lett. 96, 073901 (2006)

5. K.G. Makris et al. Opt. Lett. 30, 2466 (2005).
} 